\title{
A Comparison of the Central versus Peripheral Gastrointestinal Prokinetic Activity of Two Novel Ghrelin Mimetics
}

\author{
Ehsan N. Mohammadi, Claudio Pietra, Claudio Giuliano, Li Fugang, \\ and Beverley Greenwood-Van Meerveld
}

Oklahoma Center for Neuroscience (E.N.M., B.G.-V.M.), Department of Physiology (B.G.-V.M.), and VA Medical Center (B.G.-V.M.), University of Oklahoma Health Science Center, Oklahoma City, Oklahoma; HDB Biosciences Co. Ltd., Shanghai, People's Republic of China (L.F.); and Helsinn Healthcare SA, RPD Department, Lugano, Switzerland (C.P., C.G.)

Received May 21, 2018; accepted October 10, 2018

\begin{abstract}
The gastrointestinal (GI) prokinetic effects of ghrelin occur through direct peripheral effects on ghrelin receptors within the enteric nervous system and via the ghrelin receptor on the vagus nerve, which activate a centrally mediated mechanism. However, the relative contribution of peripheral versus central effects to the overall prokinetic effect of ghrelin agonists requires further investigation. Here, we investigated the central versus peripheral prokinetic effect of ghrelin by using two novel ghrelin agonists: HM01 (N'-[(1S)-1-(2,3-dichloro-4-methoxyphenyl) ethyl]-N-methyl-N-[1,3,3-trimethyl-(4R)-piperidyl]-urea $\mathrm{HCL}$ ) with high brain penetration compared with HMO2 ( $N^{\prime}-[(1 S)-1-$ (2,3-dichloro-4-methoxyphenyl)ethyl]- $N$-hydroxy- $N$-(1-methyl4-piperidinyl)-urea), a more peripherally acting ghrelin agonist. The pharmacokinetic profiles of both ghrelin agonists were evaluated after intravenous and oral administration in rats. The
\end{abstract}

efficacy of HM01 and HM02 was assessed in a rat model of postoperative ileus (POI) induced by abdominal surgery and in a rodent defecation assay. Pharmacokinetic results in our models confirmed that HM01, but not HM02, was a brain-penetrant ghrelin agonist. Administration of either HM01 or HM02 reversed the delayed upper and lower gastrointestinal transit induced by abdominal surgery to levels resembling the non-POI controls. In the defecation test, HM01, but not HM02, significantly increased the weight of fecal pellets. Our findings suggest that, in a rodent model of POI, synthetic ghrelin agonists stimulate Gl transit through a peripheral site of action. However, in the defecation assay, our data suggest that a ghrelin-mediated mechanism is located at a central site. Taken together, a ghrelin agonist with both central and peripheral prokinetic activity may show therapeutic potential to treat delayed GI transit disorders.

\section{Introduction}

Postoperative ileus (POI) is the temporary cessation of gastrointestinal (GI) motility following abdominal surgeries, such as appendectomy, bowel resection, and cholecystectomy. Symptoms of POI include anorexia, nausea, and constipation. The resumption of normal bowel motility postsurgery is a critical point in the recovery process (Condon et al., 1986; Zeinali et al., 2009). Thus, the development of therapeutics that have the ability to restore normal bowel activity following abdominal surgery would be of benefit to human health, resulting in less pain and suffering for patients while lowering the overall cost of postsurgical management and reducing hospital stays. Current therapeutic approaches to treat POI are limited to gut-specific opioid receptor antagonism and offlabel drugs, such as nonsteroidal anti-inflammatory drugs, dopamine $\mathrm{D}_{2}$ receptor antagonism, and serotonin $5-\mathrm{HT}_{4}$ receptor agonists (Greenwood-Van Meerveld, 2007; Van der

This work was supported by Helsinn Healthcare, SA, Lugano, Switzerland and Helsinn Therapeutics (US), Inc.

https://doi.org/10.1124/jpet.118.250738.
Ploeg et al., 2014). Drugs acting through ghrelin-mediated mechanisms represent an emerging target for drug development in the area of GI dysmotility.

Ghrelin is an orexigenic gut peptide secreted from the gastric mucosa that acts as an endogenous ligand to activate the growth hormone secretagogue receptor (GHSR) (Kojima et al., 1999), which is localized in the peripheral myenteric component of the enteric nervous system (Levin et al., 2005) and on extrinsic vagal cholinergic fibers innervating the central nervous system (Wang et al., 2015). Due to the expansive expression of ghrelin and its receptor, a host of biologic processes have been assigned to ghrelin, including the regulation of appetite and feeding behavior (Tolle et al., 2002; Davenport et al., 2005) as well as a stimulatory effect on GI transit, where ghrelin acts as a potent prokinetic to accelerate gastric emptying. In support of a prokinetic effect for ghrelin, preclinical evidence suggests that both ghrelin and small-molecule synthetic agonists of the GHSR1a accelerate GI transit and gastric emptying in rat models of POI induced by abdominal surgery (Trudel et al., 2002; Poitras et al., 2005; Venkova and Greenwood-Van Meerveld, 2008; Charoenthongtrakul et al., 2009; Venkova et al., 2009;

ABBREVIATIONS: GC, geometric center; GHSR, growth hormone secretagogue receptor; Gl, gastrointestinal; HM01, $N^{\prime}$-[(1S)-1-(2,3-dichloro-4methoxyphenyl)ethyl]- $N$-methyl- $N$-[1,3,3-trimethyl-(4R)-piperidyl]-urea Hydrochloride; HM02, $N^{\prime}-[(1 S)-1$-(2,3-dichloro-4-methoxyphenyl)ethyl]- $N$ hydroxy- $N$-(1-methyl-4-piperidinyl)-urea; LC-MS/MS, liquid chromatography-tandem mass spectrometry; PO, per os; POI, postoperative ileus. 
Greenwood-Van Meerveld et al., 2012). Small-molecule-based synthetic ghrelin agonists with greater potency, efficacy, and plasma stability are under clinical investigation for the treatment of upper GI dysmotlity such as gastroparesis. Evidence also suggests that ghrelin agonists may enhance small intestinal motility (Tack et al, 2006). The GI prokinetic effects of ghrelin occur through two mechanisms: a direct peripheral effect on ghrelin receptors within the myenteric plexus or via ghrelin receptors on the vagus and pelvic nerves activating a centrally mediated mechanism. The upper GI prokinetic effects are thought to activate peripheral ghrelin receptors in the enteric nervous system, whereas the accelerated colonic transit induced by ghrelin itself or ghrelin mimetics likely occurs through a central site of action via vagal and pelvic nerves (Binn et al., 2006; Pustovit et al., 2014; Mosińska et al., 2017). However, a study in guinea pigs suggested that the contribution of peripheral enteric ghrelin receptors in the stimulation of upper GI transit is modest, and that the gastrostimulatory action of ghrelin can occur through activation of capsaicinsensitive extrinsic vago-vagal reflex pathways (Nakamura et al., 2010). Thus, despite the potential of synthetic ghrelin agonism for the treatment of patients with GI dysmotility, the relative contribution of peripheral enteric versus central effects to the prokinetic effect of ghrelin agonists requires further investigation.

The goals of the current study were to investigate, in a rodent model of POI, whether systemic administration of novel ghrelin agonists can reverse delayed gastrointestinal transit and determine whether the prokinetic activity of ghrelin occurs through a central and/or peripheral site of action. To address these experimental goals, we used two novel ghrelin agonists: HM01 ( $N^{\prime}-[(1 S)-1-(2,3-$ dichloro-4methoxyphenyl)ethyl]- $N$-methyl- $N$-[1,3,3-trimethyl-(4R)piperidyl]-urea Hydrochloride), which shows a high binding affinity to the human GHSR- $1 \mathrm{a}\left(\mathrm{K}_{\mathrm{i}}=1.42 \pm 0.36 \mathrm{nM}\right)$ and high brain penetrance, and induces propulsive colonic contractions through a central mechanism involving the stimulation of GHSR-1a receptors on neurons in the lumbosacral spinal cord (Karasawa et al., 2014; Naitou et al., 2015); and HM02 ( $N^{\prime}-$ [(1S)-1-(2,3-dichloro-4-methoxyphenyl)ethyl]- $N$-hydroxy- $N$ (1-methyl-4-piperidinyl)-urea), a more peripherally acting ghrelin agonist with low brain penetration $\left(\mathrm{K}_{\mathrm{i}}=3.75 \pm 0.52\right.$ nM; Helsinn, unpublished observations). The effects of the two novel ghrelin agonists were investigated in a rodent model of POI induced by abdominal surgery in which we assessed gastric emptying, the geometric center (GC), and the head of a radiolabeled meal to quantify upper GI transit. Furthermore, lower GI transit was measured by inserting dye into the proximal colon at the conclusion of the POI surgery and was defined as the time taken for the first appearance of the dye in the fecal pellets. Both compounds were also studied in a defecation assay in which the numbers of fecal pellets produced in a defined time were weighed following HM01 and HM02 treatment.

\section{Materials and Methods}

Animals. Experiments were performed on male Sprague-Dawley rats $(250-350 \mathrm{~g})$ purchased from Charles River Laboratories (Wilmington, MA). Rats were housed two per cage with free access to food and water at $21-23^{\circ} \mathrm{C}$ and a 12 -hour light/dark cycle within the University of Oklahoma Health Sciences Center Department of
Comparative Medicine's animal facility in Oklahoma City, OK. For the experiments involving intravenous dosing, the animals were purchased from Charles River Laboratories with indwelling catheters implanted in the right jugular vein for administration of drugs or vehicle. All animals were acclimated to facility housing for a minimum of 1 week before experimentation. The catheters were maintained patent by gently flushing with $0.3 \mathrm{ml}$ of heparinized saline every 3 to 4 days. For the assessment of colonic transit, a separate cohort of rats was purchased from Charles River Laboratories with a catheter chronically implanted into the proximal colon (1 to $2 \mathrm{~cm}$ from the cecum) for infusion of a dye marker. The colonic catheters were flushed with $0.2 \mathrm{ml}$ of saline every 3 to 4 days. All experimental procedures were approved by the University of Oklahoma Health Sciences Center Institutional Animal Care and Use Committee (protocol number 11-027-HR). Sprague-Dawley rats (250-350 g) used for the defecation model were purchased from Beijing Vital River Laboratory Animal Technology (Beijing, China), allowed to acclimate in the animal facility at HD Biosciences (Shanghai, China), and were housed with free access to food and water at $21-23^{\circ} \mathrm{C}$ and a 12 -hour light/dark cycle. The defecation animal protocol was approved by the HD Biosciences Institutional Animal Care and Use Committee (protocol number 111).

Pharmacokinetics and Bioanalytical. Evaluation of the pharmacokinetics of HM01 and HM02 was performed in plasma after single intravenous ( 3 and $10 \mathrm{mg} / \mathrm{kg}$ i.v.) or oral [3,10, and $30 \mathrm{mg} / \mathrm{kg}$ per os (PO)] administrations to male Sprague-Dawley rats (three animals/ route/dose). Additionally, in a satellite group treated at the highest intravenous dose, HM01 and HM02 concentrations in the brain were determined at 1, 2, and 8 hours (three animals/time point). Three days before dosing and while under anesthesia (an aqueous mixture of ketamine, xylazine, and acepromazine, subcutaneously administered), the rats were surgically prepared for serial blood sample collection. The animals were fitted with a flexible 3F-polyurethane cannula (Biomedica Greto, Rome, Italy; method of sterilization: ethylene oxide) implanted in the superior vena cava via the jugular vein. At the established times (i.v.: 5, 15, 30 minutes, 1, 2, 4, 8, and 24 hours; PO: 15, 30 minutes, $1,2,4,8$, and 24 hours), $0.25 \mathrm{ml}$ of blood was taken from the cannula using syringes with anticoagulant $(7.5 \%$ EDTA solution for hematologic tests) and transferred immediately into tubes that had been precooled in an ice/water bath. Samples were centrifuged within 30 minutes at $10,000 \mathrm{~g}$ for 3 minutes at $4^{\circ} \mathrm{C}$. All plasma samples were stored at $-20^{\circ} \mathrm{C}$ pending analysis. At the established times (1, 2, and 8 hours after dosing), brains were collected, washed with $10 \mathrm{ml}$ of $0.9 \%$ sodium chloride solution, slightly dried on a blotting paper, then frozen over liquid nitrogen and stored at $-20^{\circ} \mathrm{C}$ pending analysis.

Extraction Method (Plasma). Aliquots of $25 \mu \mathrm{l}$ of rat plasma were added with $300 \mu \mathrm{l}$ of acetonitrile in a 96 -well plate containing $25 \mathrm{ng} / \mathrm{ml}$ of stable labeled internal standard (HM01-d6 or HM02-d6). The plate was vortex mixed for 5 minutes and centrifuged at $1500 \mathrm{~g}$ and $4^{\circ} \mathrm{C}$ for 15 minutes. A robotic handling station, Evolution P3 (Andrew Alliance, Geneva, Boston), was used to transfer $100 \mu \mathrm{l}$ of supernatant into a fresh 96 -well plate. Each well was added with $100 \mu \mathrm{l}$ of $10 \mathrm{mM}$ ammonium formate, $\mathrm{pH} 3.5$, and after gentle mixing of the plate, aliquots of $10 \mu \mathrm{l}$ were injected into the liquid chromatography-tandem mass spectrometry (LC-MS/MS) system.

Extraction Method (Brain). Approximately $1 \mathrm{~g}$ of brain tissue was diluted 10-fold with Dulbecco's buffered solution and sonicated using an ultrasonic processor (high-intensity ultrasonic processor; Sonics and Materials, Newtown, CT), keeping the sample on an ice bath for 40 seconds. An aliquot of $50 \mu \mathrm{l}$ of the homogenized tissue was added with $400 \mu \mathrm{l}$ of acetonitrile containing $25 \mathrm{ng} / \mathrm{ml}$ of stable labeled internal standard in a round 96 -well plate. After capping and vortex mixing, the plate was centrifuged for 15 minutes at $1500 \mathrm{~g}$ and $6^{\circ} \mathrm{C}$. A robotic handling station Evolution P3 was used to transfer $100 \mu \mathrm{l}$ of supernatant into a fresh 96 -well plate. To each well, $100 \mu \mathrm{l}$ of $10 \mathrm{mM}$ ammonium formate ( $\mathrm{pH} 3.5$ ) was added, and after centrifugation for 5 minutes at $1500 \mathrm{~g}$ and $6^{\circ} \mathrm{C}, 10-\mu \mathrm{l}$ aliquots were injected into the 
<smiles>COc1ccc([C@@H](C)NC(=O)N(C)[C@@H]2CCN(C)CC2(C)C)c(Cl)c1Cl</smiles>

B<smiles>COc1ccc([C@H](C)NC(=O)N(O)C2CCN(C)CC2)c(Cl)c1Cl</smiles>

Fig. 1. Chemical structure of ghrelin agonists HM01 (A) and HM02 (B).

LC-MS/MS system. The LC-MS/MS apparatus consisted of an Agilent 1100 high-performance liquid chromatographer with a CTC PAL autosampler (Agilent Technologies, Santa Clara, CA) and an API 3000 Mass Spectrometer (Applied Biosystems, Foster City, CA). The ionization source was a Turbo Ion Spray in positive ion mode (Harvard Apparatus, Hilliston, MA). The Multiple Reaction Monitoring transition used was $402.2 \rightarrow 126.1 \mathrm{~m} / \mathrm{z}$ for HM01, $408.2 \rightarrow 126.2 \mathrm{~m} / \mathrm{z}$ for HM01$\mathrm{d} 6,376.1 \rightarrow 131.1 \mathrm{~m} / \mathrm{z}$ for HM02, and $382.2 \rightarrow 131.1 \mathrm{~m} / \mathrm{z}$ for HM02-d6. Chromatography was performed with a Zorbax SB-C18 column $(2.1 \times$ $50 \mathrm{~mm}, 3.5 \mu \mathrm{m}$; ZefSci, Medford, MA) using a mobile phase of $10 \mathrm{mM}$ ammonium formate ( $\mathrm{pH}$ 3.5) (phase A) and acetonitrile (phase B) under gradient elution at a flow rate of $0.3 \mathrm{ml} / \mathrm{min}$. The lower limits of quantification were $5 \mathrm{ng} / \mathrm{ml}$ in plasma and $50 \mathrm{ng} / \mathrm{g}$ in brain. The calculation of pharmacokinetic parameters was performed by using the Watson package (version 7.4; Thermo Fisher Scientific, Waltham, MA) by a standard noncompartmental approach.

POI Surgery. POI was induced by a surgical procedure described as "running of the bowel" (Kalff et al., 1998). Rats were fasted overnight and anesthetized with isoflurane (5\%). The abdomen was shaved and the area was treated with alcohol and then a Betadine (povidone-iodine; Webster Vet Supplies Inc., Bessemer, AL) antiseptic for disinfection. A midline incision was made to expose the viscera. Two saline-soaked cotton-tipped applicators were used to exteriorize the small intestine and the cecum, and rats were gently massaged from the stomach to distal colon for 5 minutes. Upon completion of this procedure, the GI organs were covered with saline-soaked gauze for an additional 10 minutes. At the end of the surgery, the small intestine and the cecum were gently returned to the abdominal cavity and the incision was closed with silk sutures. The surgical procedure lasted no more than 30 minutes.
Evaluation of Gastric Motility and Intestinal Transit. An intragastric gavage of $1.5 \mathrm{ml}$ of ${ }^{99 \mathrm{~m}} \mathrm{Tc}$-labeled methylcellulose in distilled water was administered in fully conscious rats following the POI surgery. ${ }^{99 \mathrm{~m}} \mathrm{Tc}$ radioactivity was adjusted to approximately $100,000 \mathrm{cpm}$. The animals were placed in a wire-bottom cage without access to food and water. Following a 15-minute period, rats were euthanized by $\mathrm{CO}_{2}$ inhalation. The abdomen was opened and the stomach was clamped with a single silk ligature at the gastroesophageal junction and two parallel silk ligatures at the pyloric-duodenal junction. The stomach and small intestine were carefully removed from the abdominal cavity, and the total length of the small intestine was recorded. The stomach was isolated and gastric emptying was assessed via the amount of radioactivity remaining in the stomach. The small intestine was then ligated every $10 \mathrm{~cm}$, clamped, and separated. The small intestinal transit was assessed by the geometric center calculated as a function of the amount of radioactive content transported to each segment along the intestine. Calculations were made according to the method of Miller et al. (1981): geometric center $=\sum$ (percentage of total radioactivity per segment $\times$ number of segment)/100. The small intestinal transit was also characterized by the maximal distance (centimeters) reached by the head of the meal along the length of the small intestine, measured as the most distal 10 -cm segment with activity greater than $3 \times$ background activity. The radioactivity remaining in the stomach and the intestinal segments, which were arranged from proximal to distal, was measured using a gamma counter (Packard Cobra II; GMI, Ramsey, MN).

Evaluation of Colonic Transit. To evaluate colonic transit, $200 \mu \mathrm{l}$ of a nonabsorbable dye marker (trypan blue in saline) was injected into the proximal colon at the end of the surgical procedure. The abdomen was closed, and the rats were placed in a clean home cage supplied with food and water. Colonic transit time was evaluated as the period between the end of surgery and the appearance of dye in the fecal pellet. Animals not subjected to surgery and drug or vehicle treatment served as the control group. The control rats were equipped with chronically indwelling colonic catheters used to infuse the dye marker.

Defecation Model. All rats were fasted for 7 hours prior to dosing in a dark-phase model with free access to water. Immediately after administration of test compounds or vehicle (Carboxymethylcellulose, $0.5 \%$ ), the fecal pellets produced were weighed and recorded at 30 -minute intervals for 2 hours. The results were recorded as the weight of fecal pellets at each time point.

Test Compounds. The test compounds HM01 and HM02 are novel ghrelin mimetics (Fig. 1). Both test compounds were provided by Helsinn Healthcare (Lugano, Switzerland) and were stored at $4^{\circ} \mathrm{C}$ until prepared for dosing. Preceding surgery, rats were predosed with HM01 and HM02 (1 hour predose PO, 15 minutes predose i.v.). Methylcellulose and saline served as vehicle for PO and i.v.,

TABLE 1

Plasma pharmacokinetic parameters of HM01

Plasma pharmacokinetics of HM01 when dosed intravenously at 3 and $10 \mathrm{mg} / \mathrm{kg}$ or orally at 3,10, and $30 \mathrm{mg} / \mathrm{kg}$. HM01 when dosed intravenously was quickly cleared from systemic circulation and dispersed into the organs and tissues. The bioavailability after oral administration of HM01 exceeded $80 \%(n=3)$. Data are expressed as the mean \pm S.D.

\begin{tabular}{|c|c|c|c|c|c|c|}
\hline \multirow{2}{*}{ Parameter } & \multirow{2}{*}{ Units } & \multicolumn{2}{|c|}{ Intravenous } & \multicolumn{3}{|c|}{ Oral } \\
\hline & & $3 \mathrm{mg} / \mathrm{kg}$ & $10 \mathrm{mg} / \mathrm{kg}$ & $3 \mathrm{mg} / \mathrm{kg}$ & $10 \mathrm{mg} / \mathrm{kg}$ & $30 \mathrm{mg} / \mathrm{kg}$ \\
\hline $\mathrm{C}_{0083} / \mathrm{C}_{\max }$ & $\mathrm{ng} / \mathrm{ml}$ & $682 \pm 59$ & $2360 \pm 460$ & $181 \pm 44$ & $585 \pm 175$ & $1430 \pm 208$ \\
\hline$t_{\max }$ & $\mathrm{h}$ & - & - & $1.7 \pm 0.6$ & $1.7 \pm 2.0$ & $1.7 \pm 0.6$ \\
\hline $\mathrm{AUC}_{\text {last }}$ & $\mathrm{ng} \cdot \mathrm{h} / \mathrm{ml}$ & $937 \pm 145$ & $3540 \pm 765$ & $780 \pm 155$ & $3400 \pm 1010$ & $12,300 \pm 1400$ \\
\hline$t_{1 / 2, z}$ & $\mathrm{~h}$ & $1.9 \pm 0.1$ & $1.8 \pm 0.2$ & $2.6 \pm 0.2$ & $3.5 \pm 0.1$ & $3.3 \pm 0.2$ \\
\hline CL & $\mathrm{ml} / \mathrm{min} / \mathrm{kg}$ & $52 \pm 9$ & $47 \pm 11$ & - & - & - \\
\hline $\mathrm{V}_{\mathrm{ss}}$ & $\mathrm{ml} / \mathrm{kg}$ & $6600 \pm 491$ & $5910 \pm 896$ & - & - & - \\
\hline $\mathrm{V}_{\mathrm{z}}$ & $\mathrm{ml} / \mathrm{kg}$ & $8570 \pm 953$ & $7250 \pm 1090$ & - & - & - \\
\hline $\mathrm{F}$ & $\%$ & - & - & $83 \pm 21$ & $96 \pm 35$ & - \\
\hline
\end{tabular}

-, Non significant; $\mathrm{AUC}_{\text {last}}$, area under the plasma concertation curve until the last measurable concentration; CL, total clearance from the plasma; $\mathrm{C}_{\mathrm{max}}$, peak plasma

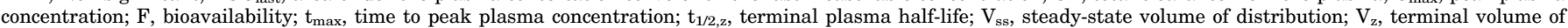
distribution. 
TABLE 2

Plasma pharmacokinetic parameters of HM02

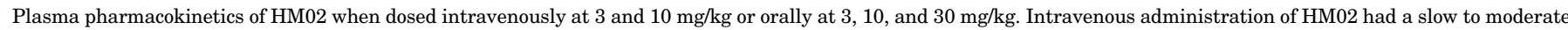
clearance from the systemic circulation. HM02 when dosed orally had a high bioavailability that exceeded $80 \%(n=3)$. Data are expressed as the mean \pm S.D.

\begin{tabular}{|c|c|c|c|c|c|c|}
\hline \multirow{2}{*}{ Parameter } & \multirow{2}{*}{ Units } & \multicolumn{2}{|c|}{ Intravenous } & \multicolumn{3}{|c|}{ Oral } \\
\hline & & $3 \mathrm{mg} / \mathrm{kg}$ & $10 \mathrm{mg} / \mathrm{kg}$ & $3 \mathrm{mg} / \mathrm{kg}$ & $10 \mathrm{mg} / \mathrm{kg}$ & $30 \mathrm{mg} / \mathrm{kg}$ \\
\hline $\mathrm{C}_{0083} / \mathrm{C}_{\max }$ & $\mathrm{ng} / \mathrm{ml}$ & $1220 \pm 117$ & $2850 \pm 214$ & $331 \pm 184$ & $925 \pm 234$ & $2580 \pm 681$ \\
\hline$t_{\max }$ & $\mathrm{h}$ & - & - & $2.00 \pm 0.00$ & $1.67 \pm 0.577$ & $2.00 \pm 0.00$ \\
\hline $\mathrm{AUC}_{\text {last }}$ & $\mathrm{ng} \cdot \mathrm{h} / \mathrm{ml}$ & $2170 \pm 197$ & $5040 \pm 281$ & $1750 \pm 727$ & $6060 \pm 669$ & $18,100 \pm 1590$ \\
\hline$t_{1 / 2, z}$ & $\mathrm{~h}$ & $3.51 \pm 1.24$ & $2.84 \pm 0.387$ & $3.01 \pm 0.516^{a}$ & $3.90 \pm 0.722$ & $2.87 \pm 0.205$ \\
\hline CL & $\mathrm{ml} / \mathrm{min} / \mathrm{kg}$ & $19.2 \pm 1.73$ & $28.3 \pm 0.666$ & - & - & - \\
\hline $\mathrm{V}_{\mathrm{ss}}$ & $\mathrm{ml} / \mathrm{kg}$ & $4790 \pm 1040$ & $6310 \pm 943$ & - & - & - \\
\hline$V_{z}$ & $\mathrm{ml} / \mathrm{kg}$ & $5790 \pm 1800$ & $6970 \pm 1030$ & - & - & - \\
\hline $\mathrm{F}^{\mathrm{z}}$ & $\%$ & - & - & $81 \pm 34$ & $120 \pm 15$ & - \\
\hline
\end{tabular}

-, Non significant; $\mathrm{AUC}_{\text {last}}$, area under the plasma concertation curve until the last measurable concentration; CL, total clearance from the plasma; $\mathrm{C}_{\text {max }}$, peak plasma

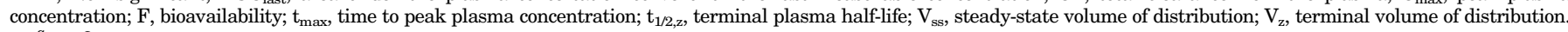

${ }^{a} n=2$.

respectively. The drug suspension was prepared so that each rat received an oral dosing volume of $0.7-1.5 \mathrm{ml}$ (dependent on body weight) for a dose of $10 \mathrm{mg} / \mathrm{kg}$, and an intravenous dosing of $0.25-0.5 \mathrm{ml}$ (dependent on body weight) for a dose of $1.4 \mu \mathrm{g} / \mathrm{kg}$. Compounds were prepared fresh on the day of use.

Data Analysis. Data are shown as the mean \pm S.D. or S.E.M., as indicated. To determine statistical significance between control and treatment groups, data were compared using one-way analysis of variance followed by a Bonferroni post-test. Results were deemed significant when $P$ values were less than 0.05 (GraphPad Prism 6.0c; GraphPad Software, La Jolla, CA). The number of animals for each experimental group was determined based on power analysis. Each set of experiments was randomized, and the persons performing experiments were not blind to the treatment to minimize the number of animals used in the studies. This approach ensured that the animals were properly acclimated to the experimenter, which is vital in preventing exposure of animals to novel stressors.

\section{Results}

Pharmacokinetic Profiles of HM01 and HM02. HM01 was rapidly cleared from the systemic circulation and distributed into tissues and organs after intravenous dosing (Tables 1 and 2). After single oral administration at doses of 3 and $10 \mathrm{mg} / \mathrm{kg}$, the bioavailability of HM01 was high and exceeded $80 \%$. After single 3 and $10 \mathrm{mg} / \mathrm{kg}$ i.v. administration of HM02, the compound was cleared from the systemic circulation with low-moderate clearance and distributed into tissues and organs. After single oral administration at doses of 3 and $10 \mathrm{mg} / \mathrm{kg}$, the bioavailability of HM02 was high and exceeded $80 \%$. The concentrations of HM01 measured in the brain at $10 \mathrm{mg} / \mathrm{kg}$ at 1,2 , and 8 hours postdosing were 1.5- to 1.9-fold higher than those in plasma. The concentrations of HM02 measured in the brain at $10 \mathrm{mg} / \mathrm{kg}$ at 1,2 , and 8 hours postdosing were lower than the corresponding values in plasma: the brain-to-plasma ratio ranged from 0.2 to 0.6. Pharmacokinetic curves of plasma and brain show the higher brain penetration of HM01 compared with HM02 (Fig. 2).

HMO1 and HM02 Reversed Delayed GI Intestinal Transit Induced by Abdominal Surgery. In control rats, we found that there was a significant delay in gastric emptying following abdominal surgery as measured by the increased amount of radioactivity remaining in the stomach in vehicle-treated rats compared with control non-POI rats $[\mathrm{F}(2,17)=9.964, P<0.001]$ (Fig. 3A). In addition, abdominal surgery produced a significant slowing of small intestinal transit quantified as a decrease in geometric center $[\mathrm{F}(2,17)=$ 14.85, $P<0.0001$ ] (Fig. 3B) and a reduction in the distance traveled by the head of the radiolabeled meal $[\mathrm{F}(2,17)=24.63$, $P<0.001$ ] (Fig. 3C). In another cohort of rats, we compared the effects of PO versus intravenous administration of HM01

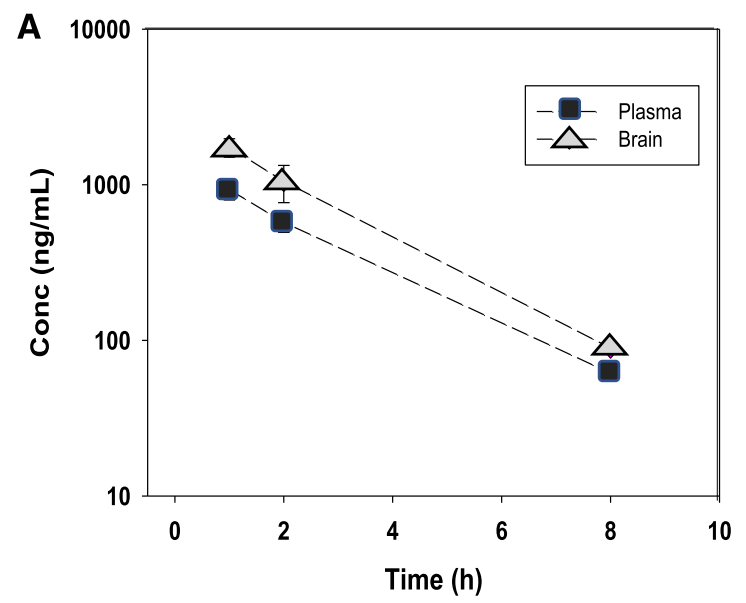

B

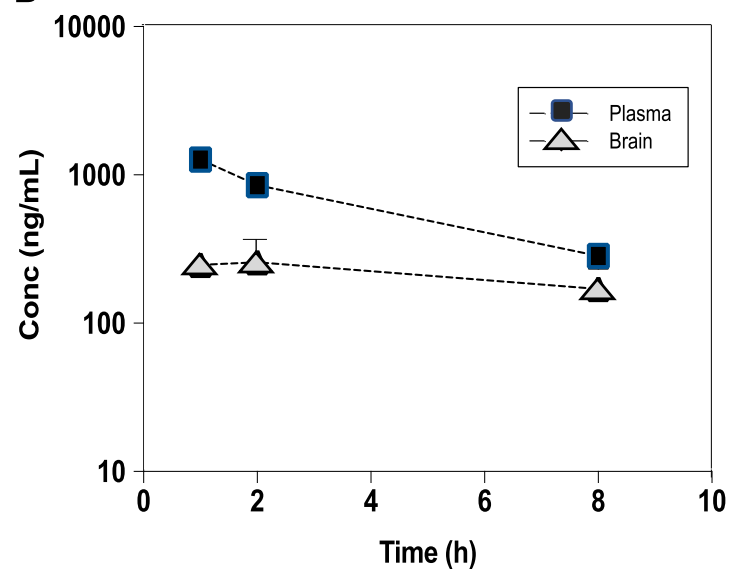

Fig. 2. Comparison of the concentration (Conc) of HM01 and HM02 in blood plasma and the brain post single intravenous administration at $10 \mathrm{mg} / \mathrm{kg}$. (A) HM01 at 1, 2, and 8 hours postadministration had a 1.5- to 2 -fold higher concentration in plasma when compared with the brain $(n=3)$. (B) HM02 at 1,2 , and 8 hours postadministration had a higher concentration in plasma when compared with the brain $(n=3)$. However, pharmacokinetic curves of plasma and brain show the higher brain penetration of HM01 compared with HM02. Data are expressed as the mean \pm S.E.M. 


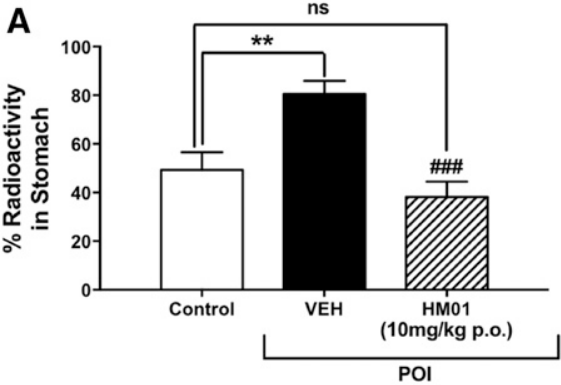

B

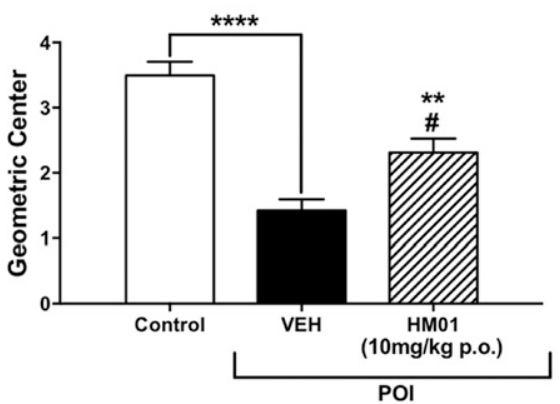

C

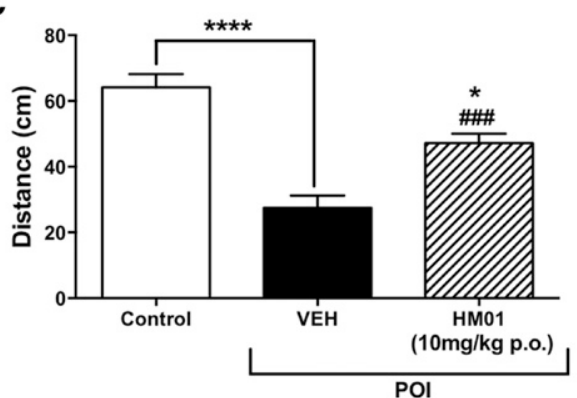

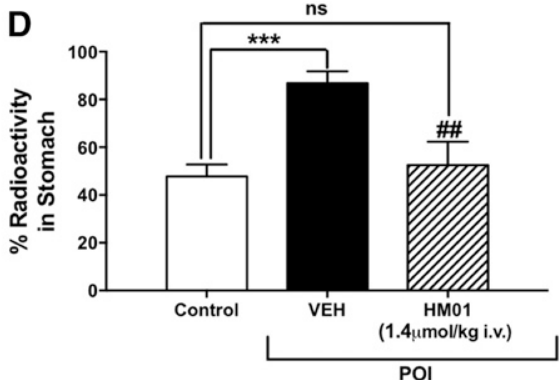

E

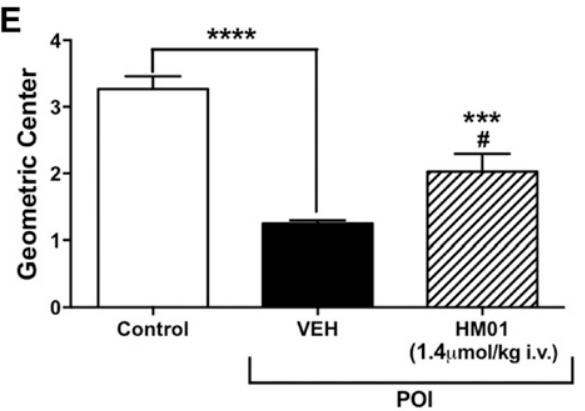

$\mathbf{F}$

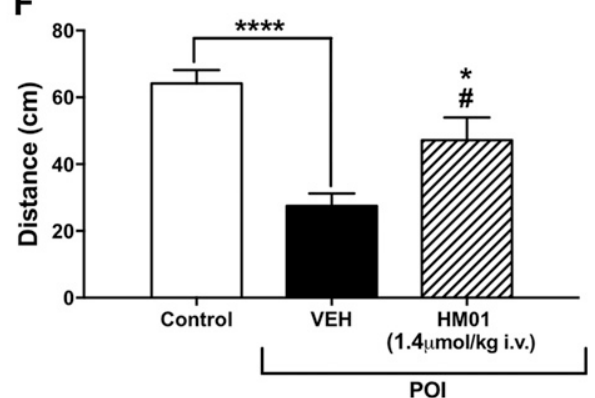

Fig. 3. Effect of HM01 on upper gastrointestinal transit in a rat model of POI (A-F) POI vehicle-treated (VEH) rats $(n=$ 6 to 7) increased the amount of radioactivity remaining in the stomach, decreased the geometric center, and decreased the distance traveled by the head of the radio labeled meal when compared with control rats $(n=6)$. HM01 $(n=8)$, when administered orally (p.o.) at $10 \mathrm{mg} / \mathrm{kg}$, decreased the amount of radioactivity remaining in the stomach to levels not statistically different from nonsurgery control rats $(P=0.53$, Bonferroni post-test) (A), increased the geometric center $(\mathrm{B})$, and increased distance traveled by the head of the meal (C). HM01 $(n=7)$, when administered intravenously at $1.4 \mu \mathrm{mol} / \mathrm{kg}$, decreased the amount of radioactivity remaining in the stomach to levels not statistically different from nonsurgery control rats $(P=0.53$, Bonferroni post-test) (D), increased the geometric center (E), and increased distance traveled by the head of the meal (F). Data shown are the mean \pm S.E.M. Statistical significance was assessed by one-way analysis of variance followed by Bonferroni post-test for multiple comparisons. $* P<0.05,{ }^{*} * P<0.01, * * * P<0.001$, and $* * * * P<0.0001$ compared with control; ${ }^{\#} P<0.05,{ }^{\# \#} P<0.01$, and ${ }^{\# \# \#} P<0.001$ compared with VEH. ns, not significant. on the delay of gastric emptying and small intestinal transit induced by abdominal surgery. Following oral or systemic administration, HM01 significantly decreased the amount of radioactivity remaining in the stomach $[\mathrm{F}(2,17)=10.4, P<$ 0.001] (Fig. 3, A and D) to a level not statistically different from that observed in nonsurgery control rats $(P=0.53$, Bonferroni post-test) and significantly increased $\mathrm{GC}[\mathrm{F}(2,17)=$ 21.59, $P<0.0001$ ] (Fig. 3, B and E) and the distance traveled by the head of the radiolabeled meal $[\mathrm{F}(2,17)=26.42, P<$ 0.001] (Fig. 3, C and F). In additional experiments, we investigated whether HM02 had any effect on POI-induced abdominal surgery. As illustrated in Fig. 4, oral and intravenous administration of HM02 significantly decreased the amount of radioactivity remaining in the stomach $[\mathrm{F}(2$, $17)=24.13, P<0.0001$ ] (Fig. 4, A and D) and increased the $\mathrm{GC}[\mathrm{F}(2,17)=19.02, P<0.0001]$ (Fig. 4 , B and E) to a level not statistically different from that observed in nonsurgery control rats ( $P=0.52$, Bonferroni post-test). Oral administration of HM02 also reversed the distance traveled by the head of the meal $[\mathrm{F}(2,17)=22.55, P<0.000]$ (Fig. 4C). After demonstrating that both HM01 and HM02 were capable of reversing the delay in gastric and small intestinal transit induced by abdominal surgery in a rodent model of POI, we advanced these observations by investigating the effect of each ghrelin agonist on the delay in colonic transit induced by abdominal surgery. In control rats, the time to the first pellet was significantly increased in POI rats treated with vehicle compared with nontreated controls (Fig. 5). Administration of either HM01 $[\mathrm{F}(2,14)=19.5, P<0.0001]$ (Fig. 5A) or HM02 $[\mathrm{F}(2,14)=34.07, P<0.0001]$ (Fig. 5B) reversed the delay in colonic transit as demonstrated by a decrease in the time to the first fecal pellet to a level not statistically different from that observed in nonsurgery control rats (HM01: $P=0.29$, HM02: $P=0.57$, Bonferroni post-test).

Effect of HMO1 and HM02 of Defecation Model. HM01 showed a dose-responsive increase in the weight of feces compared with vehicle-treated rats 30 minutes after administration $(P<0.01$ vs. vehicle at 10 and $30 \mathrm{mg} / \mathrm{kg}$ oral) (Fig. 6A), which was maintained at 24 hours post HM01 administration $(P<0.05$ vs. vehicle at 10 and $30 \mathrm{mg} / \mathrm{kg}$ oral) (Fig. 6B). As illustrated in Fig. 6C, HM01 administration significantly increased food intake at 0.1-30 mg/kg ( $P<0.01$ vs. vehicle). Conversely, HM02 showed a modest, albeit significant $(P<0.01 \mathrm{vs}$. vehicle), increase in weight of feces at 30 minutes (Fig. 7A), but no effect was observed in the 24-hour total fecal weight (Fig. 7B). In addition, HM02 significantly increased food intake at 10 and $30 \mathrm{mg} / \mathrm{kg}(P<0.01 \mathrm{vs}$. vehicle at 10 and $30 \mathrm{mg} / \mathrm{kg}$ oral) (Fig. 7C). 


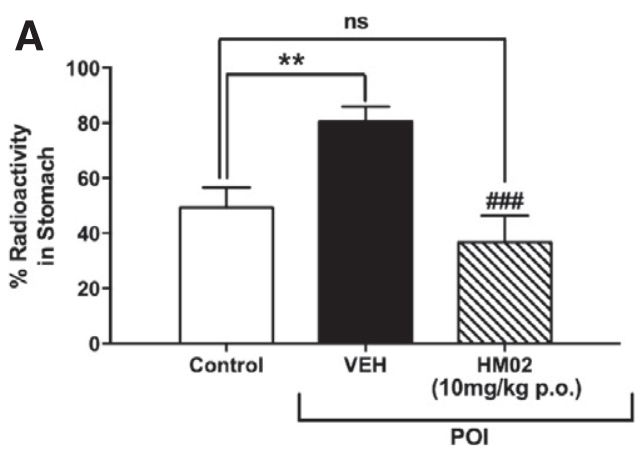

B

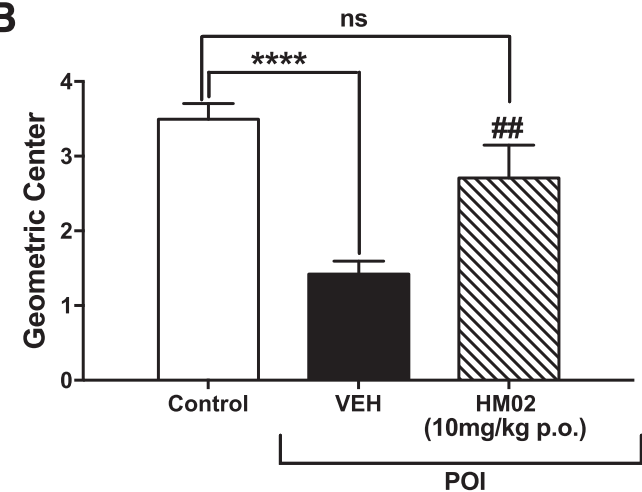

C

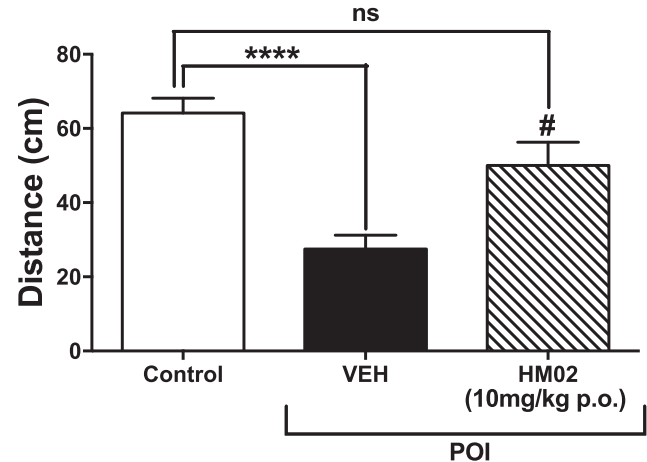

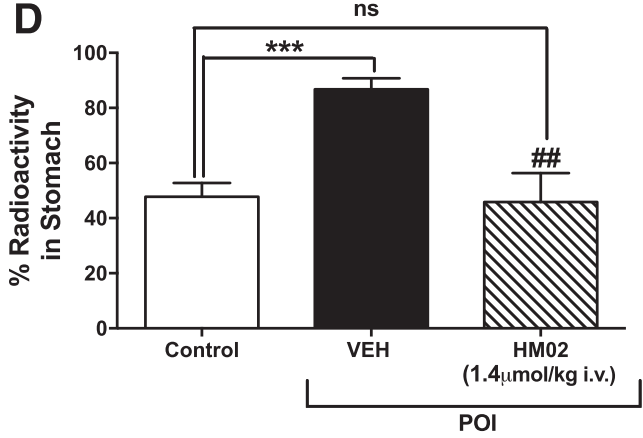

E

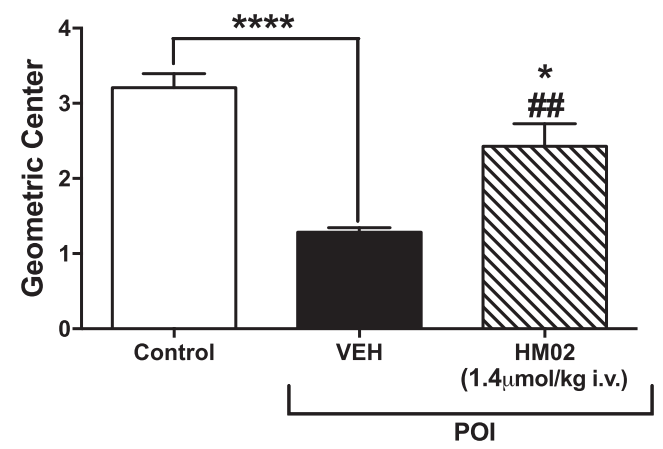

F

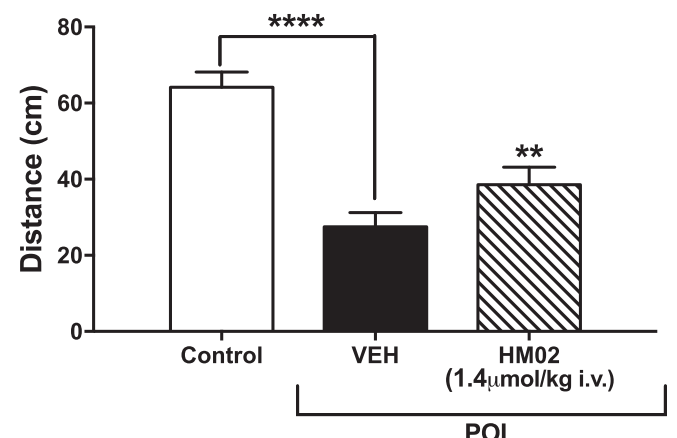

Fig. 4. Effect of HMO2 on upper gastrointestinal transit in a rat model of POI. (A-F) POI in vehicle-treated (VEH) rats $(n=6$ to 7$)$ increased the amount of radioactivity remaining in the stomach, decreased the geometric center, and decreased the distance traveled by the head of the radiolabeled meal when compared with control rats $(n=6)$. HM02 $(n=7)$ when administered orally (p.o.) at $10 \mathrm{mg} / \mathrm{kg}$ decreased the amount of radioactivity remaining in the stomach to levels not statistically different from nonsurgery control rats $(P=0.52$, Bonferroni post-test) $(\mathrm{A})$, increased the geometric center (B), and increased distance traveled by the head of the meal (C). HM02 $(n=7)$ when administered intravenously at $1.4 \mu \mathrm{mol} / \mathrm{kg}$ decreased the amount of radioactivity remaining in the stomach to levels not statistically different from nonsurgery control rats $(P=0.52$, Bonferroni post-test) (D), increased the geometric center $(\mathrm{E})$, and had no effect on distance traveled by the head of the meal $(\mathrm{F})$. Data shown are the mean \pm S.E.M. Statistical significance was assessed by one-way analysis of variance followed by Bonferroni post-test for multiple comparisons. $* P<0.05, * * P<0.01$, $* * * P<0.001$, and **** $P<0.0001$ compared with control; ${ }^{\#} P<0.05$, ${ }^{\# \#} P<0.01$, and ${ }^{\# \# \#} P<0.001$ compared with VEH. ns, not significant.

\section{Discussion}

Ghrelin is an orexigenic hormone produced within endocrine cells of the gastric mucosa that has previously been shown to promote propulsive motility in the stomach and small intestine through activation of ghrelin receptors expressed in the upper GI tract (Wang et al., 2015). Emerging clinical evidence suggests that ghrelin mimetics may prove useful in the treatment of upper GI hypomotility disorders, including diabetic gastroparesis and postoperative ileus (Avau et al., 2013). In the present study, we demonstrated that surgical manipulation of the bowel in a rodent model caused a delay of gastric emptying, decreased the geometric center, reduced the distance traveled by the head of the radioactive meal, and increased the time to first fecal pellet output. These results are consistent with our previous studies and confirm that this model of delayed GI transit in rats following surgery is an effective experimental tool to investigate the efficacy of new therapeutic treatments relevant to the postsurgical GI dysfunction apparent in patients (Venkova et al., 2009). Using this experimental model, we aimed to investigate whether the GI prokinetic effects of ghrelin agonism occur through enteric activation and/or via centrally mediated mechanisms. We found that, following systemic administration of either HM01, a ghrelin agonist that crosses the blood-brain barrier, or HM02, a peripherally restricted 

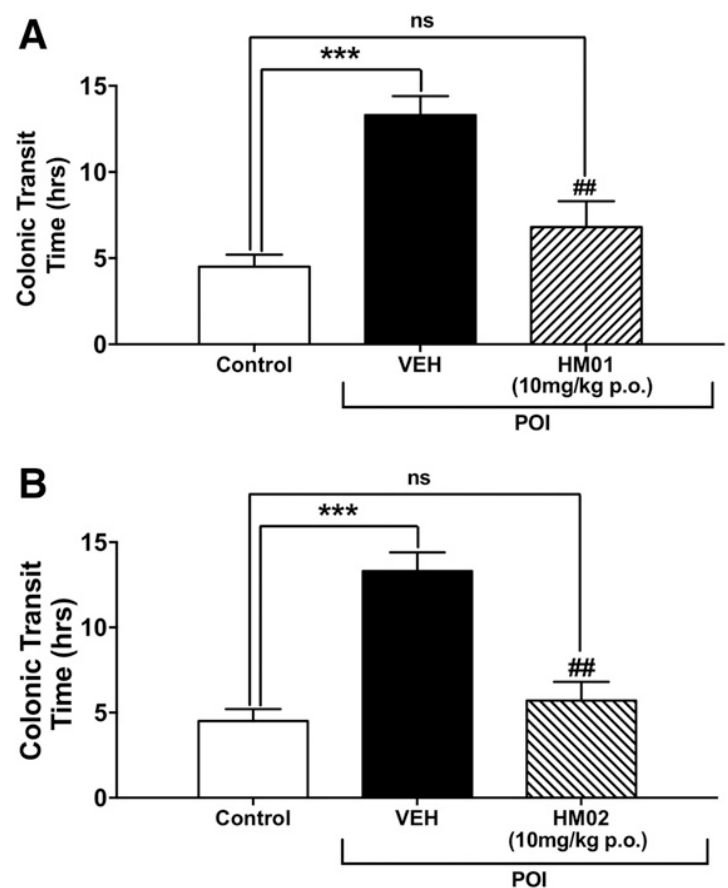

Fig. 5. Effect of HM01 and HM02 on lower gastrointestinal transit in a rat model of POI. (A and B) POI in vehicle-treated (VEH) rats $(n=7)$ significantly increased colonic transit time. HM01 $(n=8)(\mathrm{A})$ and HM02 $(n=7)$ (B), when administered orally (p.o.) at $10 \mathrm{mg} / \mathrm{kg}$, significantly decreased colonic transit time to a level not statistically different from that observed in nonsurgery control rats (HM01: $P=0.29$, HM02: $P=0.57$, Bonferroni post-test). Data shown are the mean \pm S.E.M. Statistical significance was assessed by one-way analysis of variance followed by Bonferroni post-test for multiple comparisons. ${ }^{* * *} P<0.001$ compared with control; ${ }^{\# \#} P<0.01$ compared with VEH. ns, not significant.

ghrelin agonist, there was a reversal of delayed upper and lower GI transit resembling GI transit observed in the nonPOI controls. Based upon these compelling experimental responses produced by a peripherally restricted ghrelin agonist (HM02), we conclude that the acceleration of upper and lower GI transit by ghrelin agonism occurs predominantly through a peripheral site of action, likely involving ghrelin receptor-mediated activation within the myenteric plexus. However, the activation of ghrelin receptors on vagal afferents by a peripherally restricted ghrelin agonist cannot be completely ruled out. In concert, we found that HM01 activated a centrally mediated defecation reflex to increase fecal pellet production and was superior to HM02, suggesting that a ghrelin receptor-mediated mechanism controlling distal colorectal motility may be located at a central site. However, it is possible that a ghrelin-mediated increase in food consumption may have indirectly altered 24-hour fecal pellet production. Despite convincing evidence in preclinical models and humans for the prokinetic activity of ghrelin agonism throughout the GI tract, there is conflicting evidence related to the relative roles for peripheral enteric neural versus central mechanisms contributing to the GI prokinetic effects of ghrelin. Thus, we used another novel ghrelin agonist, HM02, with low brain penetration compared with HM01 but with high affinity to the human GHSR-1a. Following systemic administration of HM02, we demonstrated a reversal of POI-induced gastroparesis and a delay in upper small intestinal transit induced by POI, which is consistent with a peripheral site of action, likely at the level of the enteric nervous system rather than a direct effect on the smooth muscle. In support, studies have shown GHSR immunoreactivity within neuronal cell bodies and fibers of the myenteric plexus in the stomach and colon isolated from rats and humans, whereas smooth muscle cells did not express GHSR immunoreactivity (Dass et al., 2003). Taken together, these data are strongly supportive of an activation of ghrelin receptors in the periphery leading to the prokinetic effect of ghrelin agonism.

The finding that ghrelin agonists, with an ability to cross the blood-brain barrier, stimulate colonic prokinetic activity points to the potential usefulness of centrally acting ghrelin agonists for the treatment of patients with chronic constipation (Shimizu et al., 2006; Shafton et al., 2009; Avau et al., 2013; Pustovit et al., 2014; Acosta et al., 2015; Mosińska et al., 2017). Thus, we investigated whether a novel ghrelin agonist with the ability to penetrate the blood-brain barrier (HM01) had any effect on delayed GI motility. Our finding that a novel ghrelin agonist with brain penetrance following oral administration reversed POI-induced gastroparesis and a delay in upper GI transit suggests that a central brainstem mechanism of action may be involved in the gastroprokinetic effects of ghrelin. In support, preclinical studies in rats have shown that prokinetic effects of ghrelin are prevented or reversed by vagotomy (Masuda et al., 2000; Asakawa et al., 2001; Fukuda et al., 2004; Nakamura et al., 2010). Moreover, centrally or peripherally administered ghrelin in rats acts as a modulator stimulating colonic motility via hypothalamic nuclei including the arcuate nucleus, dorsomedial hypothalamic nucleus, and paraventricular nucleus (Root and Root, 2002; Fujino et al., 2003; Chen and Tsai, 2012). Furthermore, administration of ghrelin directly into the paraventricular nucleus dosedependently accelerated small intestinal transit in rats that was competitively inhibited by a GHSR antagonist (Wang et al., 2015). However, in the current study, the possibility exists that despite its ability to penetrate the brain, peripheral administration of HM01 may also activate GHSRs in the myenteric plexus of the enteric nervous system to promote GI motility. Another observation in the current study, pointing to the importance of a central site of action for ghrelin agonism, was that in the defecation test, HM01, but not HM02, significantly increased the weight of fecal pellets, suggesting that a central mechanism(s) played an important role in the activation of the defecation reflex in normal healthy rats, which is consistent with previous work using other centrally acting ghrelin agonists likely acting through extrinsic pelvic cholinergic nerves (Peeters, 2003; Shimizu et al., 2006; Shafton et al., 2009; Avau et al., 2013; Pustovit et al., 2014).

Interestingly, the effect of ghrelin agonism seems to be much more pronounced on the stomach than on the small intestine. Similar results were also previously shown for ghrelin and GHRP-6 (De Winter et al., 2004; Depoortere et al., 2005). Since our current in vivo technique does not allow us to investigate the effect of ghrelin agonism on small intestinal transit in isolation, the possibility exists that the delay in small intestinal transit induced by ghrelin agonism might just result from the delayed gastric emptying delivering less of the radioactive meal to the small intestine.

In addition to studying the effect of ghrelin on delayed transit induced by abdominal surgery, experimental evidence points to the efficacy of ghrelin mimetics to accelerate GI transit in another model of delayed GI transit. In mice with diabetic gastroparesis, the peripheral administration of 


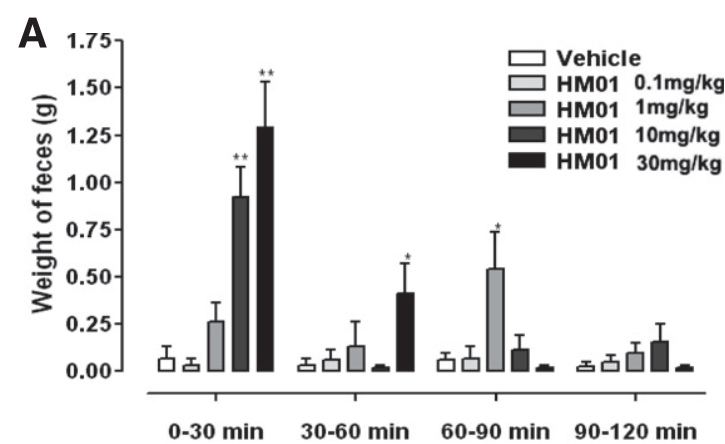

B

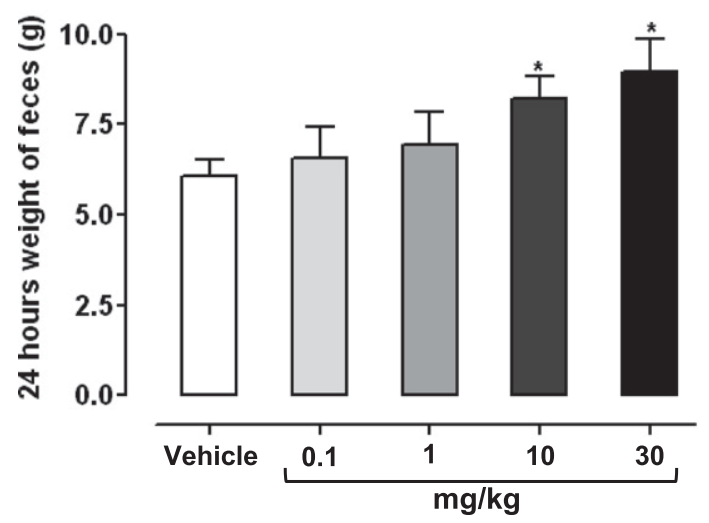

C

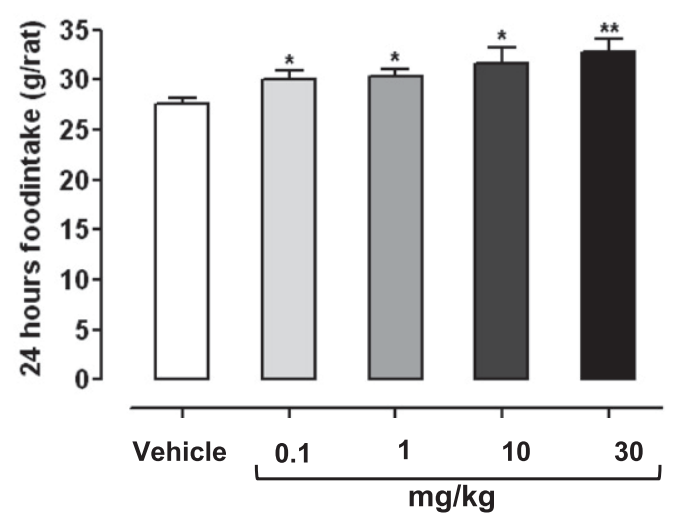

Fig. 6. Effect of HM01 in defecation test, measured as weight of feces, 24-hour fecal weight, and total food intake. HM01 $(n=6)$ significantly increased the weight of the feces (A), the 24-hour fecal weight (B), and food intake (C) in a dose-dependent manner. Data are shown as the mean \pm S.E.M. Statistical significance was assessed by one-way analysis of variance followed by Bonferroni post-test for multiple comparisons. $* P<$ 0.05 and $* * P<0.01$ compared with vehicle.

ghrelin significantly increased gastric emptying, intestinal transit, and colonic transit (Zheng et al., 2008). However, it is unclear whether these prokinetic effects are attributable to peripheral or central mechanisms. In a rat model of Parkinson's disease induced by 6-hydroxydopamine, the finding that oral administration of HM01 caused a reversal of delayed GI transit through a mechanism involving c-fos activation in selective brain and spinal areas regulating GI function demonstrates the potential of ghrelin agonism for the treatment of Parkinson's disease, possibly through a central site of action (Karasawa et al., 2014). Interestingly, repeated daily administration of HM01 for up to 8 days remained

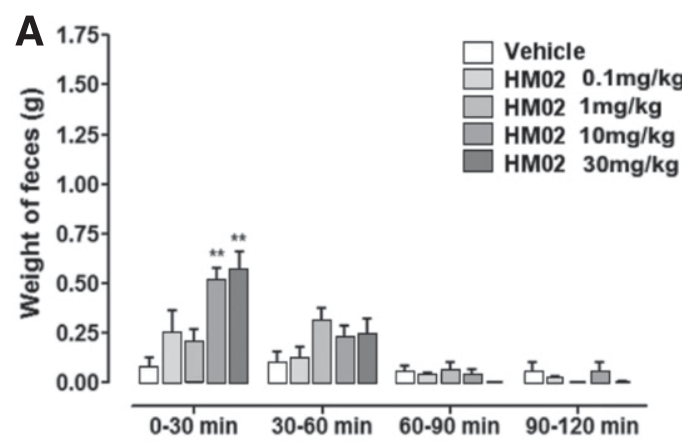

B
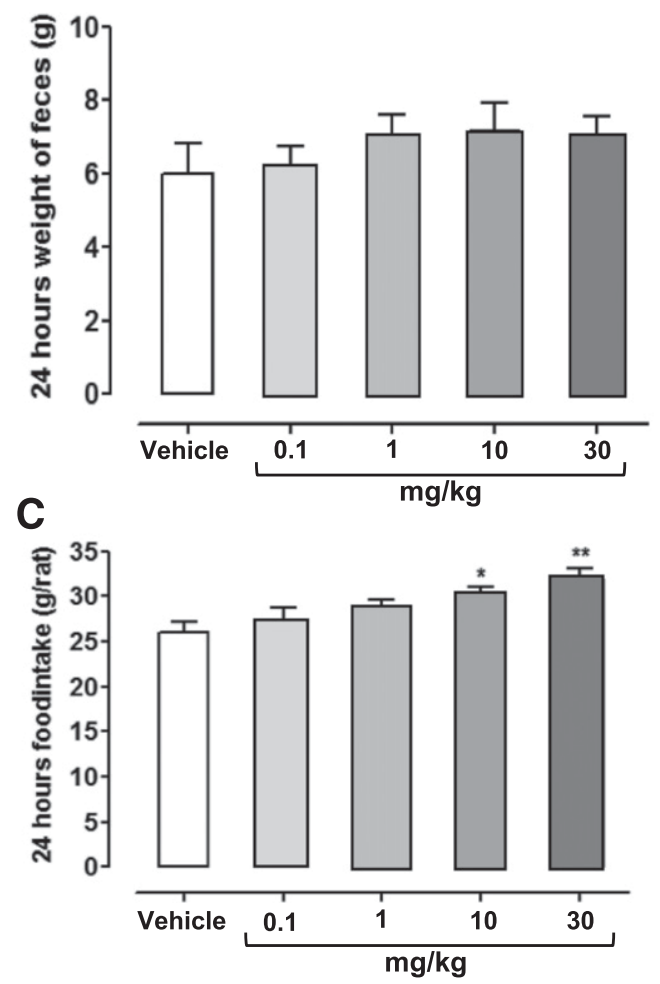

Fig. 7. Effect of HM02 in defecation test, measured as weight of feces, 24-hour fecal weight, and total food intake. HM029 $(n=6)$ significantly increased the weight of the feces (A) and food intake (C) in a dosedependent manner but had no effect on 24-hour feces weight (B). Data are shown as the mean \pm S.E.M. Statistical significance was assessed by oneway analysis of variance followed by Bonferroni post-test for multiple comparisons. $* P<0.05$ and $* * P<0.01$ compared with vehicle.

efficacious in the Parkinson's disease rodent model of colonic dysmotility (Karasawa et al., 2014).

Although the present study did not evaluate these novel ghrelin mimetics in non-POI rats, other studies have identified that ghrelin in normal rats accelerates gastric emptying (Trudel et al., 2002), colonic motility (Huang et al., 2016), and increases neuronally mediated rat-isolated forestomach contractility (Bassil et al., 2006). There is also evidence that ghrelin induces migrating motor complex-like activity in rats (Fujino et al., 2003; Edholm et al., 2004). Taken together, there is ample evidence showing the prokinetic effects of exogenous ghrelin under normal conditions. Furthermore, HM01 has been reported to induce a dose-dependent increase in propulsive contraction of the colorectum in normal rats which was prevented by spinal cord transection, thus 
suggesting a spinal site of action of ghrelin receptors (Naitou et al., 2015).

In summary, our findings suggest that in an experimental model of delayed GI transit induced by abdominal surgery, two novel synthetic ghrelin agonists-HM01, with higher central penetrance than HM02-accelerate transit in the stomach, small intestine, and colon, which strongly points to a peripheral gastroprokinetic effect in a rodent model of POI. In contrast, in the defecation assay, our finding that only HM01 increased fecal pellet output suggests that a ghrelin receptor-mediated prokinetic effect occurs through a central site. Taken together, our experimental evidence suggests that in patients with delayed gastric, small intestinal, and colonic motility, a novel ghrelin agonist, such as HM02, with low brain penetrance may prove beneficial when used acutely. However, a ghrelin agonist with the ability to cross into the central nervous system, such as HM01, could be used to treat patients with chronic constipation or defecation disorders.

\section{Authorship Contributions}

Participated in research design: Mohammadi, Pietra, Giuliano, Greenwood-Van Meerveld.

Conducted experiments: Mohammadi, Fugang.

Contributed new reagents or analytic tools: Mohammadi, Pietra, Greenwood-Van Meerveld.

Performed data analysis: Mohammadi, Pietra, Greenwood-Van Meerveld.

Wrote or contributed to the writing of the manuscript: Mohammadi, Pietra, Giuliano, Greenwood-Van Meerveld.

\section{References}

Acosta A, Camilleri M, Kolar G, Iturrino J, Szarka LA, Boldingh A, Burton D, Ryks M, Rhoten D, Zinsmeister AR, et al. (2015) Relamorelin relieves constipation and accelerates colonic transit in a phase 2 , placebo-controlled, randomized trial. Clin Gastroenterol Hepatol 13:2312-2319.e1.

Asakawa A, Inui A, Kaga T, Yuzuriha H, Nagata T, Ueno N, Makino S, Fujimiya M, Niijima A, Fujino MA, et al. (2001) Ghrelin is an appetite-stimulatory signal from stomach with structural resemblance to motilin. Gastroenterology 120:337-345.

Avau B, Carbone F, Tack J, and Depoortere I (2013) Ghrelin signaling in the gut, its physiological properties, and therapeutic potential. Neurogastroenterol Motil 25: $720-732$.

Bassil AK, Dass NB, and Sanger GJ (2006) The prokinetic-like activity of ghrelin in rat isolated stomach is mediated via cholinergic and tachykininergic motor neurones. Eur J Pharmacol 3:146-152.

Binn M, Albert C, Gougeon A, Maerki H, Coulie B, Lemoyne M, Rabasa Lhoret R, Tomasetto C, and Poitras P (2006) Ghrelin gastrokinetic action in patients with neurogenic gastroparesis. Peptides 27:1603-1606.

Charoenthongtrakul S, Giuliana D, Longo KA, Govek EK, Nolan A, Gagne S, Morgan K, Hixon J, Flynn N, Murphy BJ, et al. (2009) Enhanced gastrointestinal motility with orally active ghrelin receptor agonists. J Pharmacol Exp Ther 329:1178-1186.

Chen CY and Tsai CY (2012) Ghrelin and motilin in the gastrointestinal system. Curr Pharm Des 18:4755-4765.

Condon RE, Frantzides CT, Cowles VE, Mahoney JL, Schulte WJ, and Sarna SK (1986) Resolution of postoperative ileus in humans. Ann Surg 203:574-581.

Dass NB, Munonyara M, Bassil AK, Hervieu GJ, Osbourne S, Corcoran S, Morgan M, and Sanger GJ (2003) Growth hormone secretagogue receptors in rat and human gastrointestinal tract and the effects of ghrelin. Neuroscience 120:443-453.

Davenport AP, Bonner TI, Foord SM, Harmar AJ, Neubig RR, Pin JP, Spedding M, Kojima M, and Kangawa K (2005) International Union of Pharmacology. LVI. Ghrelin receptor nomenclature, distribution, and function. Pharmacol Rev $\mathbf{5 7}$ $541-546$

Depoortere I, De Winter B, Thijs T, De Man J, Pelckmans P, and Peeters T (2005) Comparison of the gastroprokinetic effects of ghrelin, GHRP-6 and motilin in rats in vivo and in vitro. Eur J Pharmacol 515:160-168.

De Winter BY, De Man JG, Seerden TC, Depoortere I, Herman AG, Peeters TL and Pelckmans PA (2004) Effect of ghrelin and growth hormone-releasing peptide 6 on septic ileus in mice. Neurogastroenterol Motil 16:439-446.

Edholm T, Levin F, Hellström PM, and Schmidt PT (2004) Ghrelin stimulates motility in the small intestine of rats through intrinsic cholinergic neurons. Regul Pept. 3:25-30.

Fujino K, Inui A, Asakawa A, Kihara N, Fujimura M, and Fujimiya M (2003) Ghrelin induces fasted motor activity of the gastrointestinal tract in conscious fed rats. $J$ Physiol 550:227-240

Fukuda H, Mizuta Y, Isomoto H, Takeshima F, Ohnita K, Ohba K, Omagari K, Taniyama K, and Kohno S (2004) Ghrelin enhances gastric motility through direct stimulation of intrinsic neural pathways and capsaicin-sensitive afferent neurones in rats. Scand J Gastroenterol 39:1209-1214.

Greenwood-Van Meerveld B (2007) Emerging drugs for postoperative ileus. Expert Opin Emerg Drugs 12:619-626.

Greenwood-Van Meerveld B, Tyler K, Mohammadi E, and Pietra C (2012) Efficacy of ipamorelin, a ghrelin mimetic, on gastric dysmotility in a rodent model of postoperative ileus. $J$ Exp Pharmacol 4:149-155.

Huang HH, Ting CH, Syu YF, Chang SC, and Chen CY (2016) Correlation between colonic secretion and colonic motility in rats: Role of ghrelin. World J Gastroenterol 46:10140-10147.

Kalff JC, Schraut WH, Simmons RL, and Bauer AJ (1998) Surgical manipulation of the gut elicits an intestinal muscularis inflammatory response resulting in postsurgical ileus. Ann Surg 228:652-663.

Karasawa H, Pietra C, Giuliano C, Garcia-Rubio S, Xu X, Yakabi S, Taché Y, and Wang L (2014) New ghrelin agonist, HM01 alleviates constipation and L-dopadelayed gastric emptying in 6-hydroxydopamine rat model of Parkinson's disease. Neurogastroenterol Motil 26:1771-1782.

Kojima M, Hosoda H, Date Y, Nakazato M, Matsuo H, and Kangawa K (1999) Ghrelin is a growth-hormone-releasing acylated peptide from stomach. Nature 402: 656-660.

Levin F, Edholm T, Ehrström M, Wallin B, Schmidt PT, Kirchgessner AM, Hilsted LM, Hellström PM, and Näslund E (2005) Effect of peripherally administered ghrelin on gastric emptying and acid secretion in the rat. Regul Pept 131:59-65.

Masuda Y, Tanaka T, Inomata N, Ohnuma N, Tanaka S, Itoh Z, Hosoda H, Kojima M, and Kangawa K (2000) Ghrelin stimulates gastric acid secretion and motility in rats. Biochem Biophys Res Commun 276:905-908.

Miller MS, Galligan JJ, and Burks TF (1981) Accurate measurement of intestinal transit in the rat. $J$ Pharmacol Methods 6:211-217.

Mosińska P, Zatorski H, Storr M, and Fichna J (2017) Future treatment of constipation-associated disorders: role of relamorelin and other ghrelin receptor agonists. J Neurogastroenterol Motil 23:171-179.

Naitou K, Mamerto TP, Pustovit RV, Callaghan B, Rivera LR, Chan AJ, Ringuet MT, Pietra C, and Furness JB (2015) Site and mechanism of the colokinetic action of the ghrelin receptor agonist, HM01. Neurogastroenterol Motil 27:1764-1771.

Nakamura T, Onaga T, and Kitazawa T (2010) Ghrelin stimulates gastric motility of the Guinea pig through activation of a capsaicin-sensitive neural pathway: in vivo and in vitro functional studies. Neurogastroenterol Motil 22:446-452, e107.

Peeters TL (2003) Central and peripheral mechanisms by which ghrelin regulates gut motility. J Physiol Pharmacol 54 (Suppl 4):95-103.

Poitras P, Polvino WJ, and Rocheleau B (2005) Gastrokinetic effect of ghrelin analog RC-1139 in the rat. Effect on post-operative and on morphine induced ileus. Peptides 26:1598-1601.

Pustovit RV, Callaghan B, Kosari S, Rivera LR, Thomas H, Brock JA, and Furness JB (2014) The mechanism of enhanced defecation caused by the ghrelin receptor agonist, ulimorelin. Neurogastroenterol Motil 26:264-271.

Root AW and Root MJ (2002) Clinical pharmacology of human growth hormone and its secretagogues. Curr Drug Targets Immune Endocr Metabol Disord 2:27-52.

Shafton AD, Sanger GJ, Witherington J, Brown JD, Muir A, Butler S, Abberley L, Shimizu Y, and Furness JB (2009) Oral administration of a centrally acting ghrelin receptor agonist to conscious rats triggers defecation. Neurogastroenterol Motil 21: 71-77.

Shimizu Y, Chang EC, Shafton AD, Ferens DM, Sanger GJ, Witherington J, and Furness JB (2006) Evidence that stimulation of ghrelin receptors in the spinal cord initiates propulsive activity in the colon of the rat. J Physiol 576:329-338.

Tack J, Depoortere I, Bisschops R, Delporte C, Coulie B, Meulemans A, Janssens J, and Peeters $\mathrm{T}$ (2006) Influence of ghrelin on interdigestive gastrointestinal motility in humans. Gut 55:327-333.

Tolle V, Bassant MH, Zizzari P, Poindessous-Jazat F, Tomasetto C, Epelbaum J, and Bluet-Pajot MT (2002) Ultradian rhythmicity of ghrelin secretion in relation with GH, feeding behavior, and sleep-wake patterns in rats. Endocrinology 143 1353-1361.

Trudel L, Tomasetto C, Rio MC, Bouin M, Plourde V, Eberling P, and Poitras P (2002) Ghrelin/motilin-related peptide is a potent prokinetic to reverse gastric postoperative ileus in rat. Am J Physiol Gastrointest Liver Physiol 282:G948-G952.

Van der Ploeg L, Laken H, Sharma S, Datta R, Halem H, Dong J, Touvay C, Teillot M, Noonan P, Tartaglia L, et al. (2014) Preclinical gastrointestinal prokinetic ef ficacy and endocrine effects of the ghrelin mimetic RM-131. Life Sci 109:20-29.

Venkova K and Greenwood-Van Meerveld B (2008) Application of ghrelin to gastrointestinal diseases. Curr Opin Investig Drugs 9:1103-1107.

Venkova K, Mann W, Nelson R, and Greenwood-Van Meerveld B (2009) Efficacy of ipamorelin, a novel ghrelin mimetic, in a rodent model of postoperative ileus. $J$ Pharmacol Exp Ther 329:1110-1116.

Wang Y, Chen F, Shi H, Jiang J, Li H, Qin B, and Li Y (2015) Extrinsic ghrelin in the paraventricular nucleus increases small intestinal motility in rats by activating central growth hormone secretagogue and enteric cholinergic receptors. Peptides 74:43-49.

Zeinali F, Stulberg JJ, and Delaney CP (2009) Pharmacological management of postoperative ileus. Can J Surg 52:153-157.

Zheng Q, Qiu WC, Yan J, Wang WG, Yu S, Wang ZG, and Ai KX (2008) Prokinetic effects of a ghrelin receptor agonist GHRP-6 in diabetic mice. World $J$ Gastroenterol 14:4795-4799.

Address correspondence to: Dr. Beverley Greenwood-Van Meerveld, Oklahoma Center for Neuroscience, BRC 272, 975 N.E. 10th Street, Oklahoma City, OK 73104. E-mail: Beverley-Greenwood@ouhsc.edu 